\title{
On the structure of polar faculae on the Sun
}

\author{
O. V. Okunev ${ }^{1,2}$ and F. Kneer ${ }^{1}$ \\ 1 Universitäts-Sternwarte, Geismarlandstr. 11, 37083 Göttingen, Germany \\ e-mail: [Kneer;olok]@uni-sw.gwdg.de \\ ${ }^{2}$ Central Astronomical Observatory at Pulkovo, 196140 St. Petersburg, Russia
}

Received 20 April 2004 / Accepted 25 May 2004

\begin{abstract}
Faculae on the polar caps of the Sun, in short polar faculae (PFe), are investigated. They take part in the magnetic solar cycle. Here, we study the fine structures of PFe, their magnetic fields and their dynamics on short time scales. The observations stem from several periods in 2001 and 2002. They consist of spectropolarimetric data (Stokes $I$ and $V$ ) taken in the Fe I 6301.5 and $6302.5 \AA$ and Fe II $6149.3 \AA$ lines with the Gregory-Coudé Telescope (GCT) and the Vacuum Tower Telescope (VTT) at the Observatorio del Teide on Tenerife. At the VTT, the "Göttingen" two-dimensional Fabry-Perot spectrometer was used. It allows image reconstruction with speckle methods resulting in spatial resolution of approximately 0 ' 25 for broadband images and 0 '. 5 for magnetograms. The application of singular value decomposition yielded a polarimetric detection limit of $|V| \approx 2 \times 10^{-3} I_{\mathrm{c}}$. We find that $\mathrm{PFe}$, of size of $1^{\prime \prime}$ or larger, possess substantial fine structure of both brightness and magnetic fields. The brightness and the location of polar facular points change noticeably within $50 \mathrm{~s}$. The facular points have strong, kilo-Gauss magnetic fields, they are unipolar with the same polarity as the global, poloidal magnetic field. The ambient areas, however, exhibit weak flux features of both polarities, as in the quiet Sun near disk center. Strong upflows of $1 \mathrm{~km} \mathrm{~s}^{-1}$ are detected in the intensity profiles of PFe. The zero-crossings of the Stokes $V$ profiles yield an average velocity of the magnetized plasma of $v_{\mathrm{FT}} \approx-0.4 \mathrm{~km} \mathrm{~s}^{-1}$ (towards the observer).
\end{abstract}

Key words. Sun: faculae, plages - Sun: magnetic fields - techniques: high angular resolution

\section{Introduction}

Polar faculae are bright small-scale structures near the poles of the Sun. They are seen under good seeing conditions in white light and in chromospheric lines inside the limb. Polar faculae (henceforth PFe) are an important aspect of solar magnetism (Waldmeier 1955, 1965; Sheeley 1964, 1991; Makarov et al. 2003a,b, and references in these works). Following Makarov et al., the PFe precede the sunspot cycle by 5-6 years. During sunspot minimum, they appear at the polar caps of the Sun at heliographic latitudes $\left|\phi_{\odot}\right| \geq 60^{\circ}$. During the growth of sunspot activity, this lower latitude boundary migrates towards the poles and the regions of PF appearance vanish during the maximum of sunspot activity (see, e.g., Makarov \& Sivaraman 1989; Makarov et al. 2003b). Wang \& Sheeley (2003), from numerical modelling, describe the poleward migration of the lower latitude boundary as due to emergence and growth of bipolar regions at medium latitudes during the activity cycle.

PFe are caused by magnetic fields, and it has been shown by Homann et al. (1997) that the field strengths of PFe are in the kilo-Gauss range. While Homann et al. suggest that the magnetic polarity of the PFe is that of the general polar magnetic field, Zhang \& Zhang (1999) find no preference of polarity of small-scale magnetic structures at the solar poles. DeForest et al. (1997) suggest from coordinated observations with the EIT and MDI instruments on board the SOHO spacecraft that polar coronal plumes are rooted in X-ray bright points corresponding to unipolar magnetic concentrations on network cell boundaries. Yet other authors, e.g. Wilhelm et al. (1998), argue that polar plumes have their footing in closed loop structures, and later on Wilhelm et al. (2000) identify the outflow regions with dark coronal areas, not with the bright plumes. Thus, the research into the magnetic cycle at the solar poles, the origin of the fast solar wind, and the rôle of the polar coronal plumes makes the study of PFe an important issue.

Apart from the notion of strong fields by Homann et al. (1997) little is known about the internal structure of PFe. Hence the present study aims at scrutinizing their spatial fine structure, including the fine structure of their magnetic fields, and the temporal evolution of PFe on short time scales. White light and spectropolarimetric data have been analysed with emphasis on high spatial resolution and high polarimetric sensitivity at the same time. Both are needed for a study of structure and dynamics of PFe since these are small-scale features and since the polarimetric signals from small structures at the poles of the Sun, i.e. close to the solar limb, are small. The results of the data analysis make it possible to test magnetic flux tube (MFT) models and their compatibility with observations close to the solar limb.

The next section describes the observations of PFe and the data analysis. Section 3 presents a selection of observational results and Sect. 4 concludes the paper. In a further study, we 
constructed models of small-scale MFTs, which are randomly distributed in an area corresponding to the spatial resolution element of the observations. Stokes $I$ and $V$ profiles of magnetically sensitive lines were calculated for comparison with the PF observations. The modelling and its results will be presented in a forthcoming publication. The reader is also referred to Okunev (2004), where a detailed account of the data analysis is given and more observational results and the models are described.

\section{Observations and data analysis}

The observations were obtained at different times with two telescopes, the Gregory-Coudé Telescope (GCT, dismounted in spring 2002) at the Observatorio del Teide and the Vacuum Tower Telescope (VTT) at the same observatory. The GCT allowed observations with high spectral resolution and good polarimetric sensitivity, while the spatial resolution of the GCT data was often 1", sometimes better. With the VTT, we can perform two-dimensional spectropolarimetry such that seeing effects can be reduced by standard methods of speckle reconstruction yielding a spatial resolution of the order of 0.5 in the polarimetric data.

In the following, we will give - relatively short - accounts of the observations and the data analyses, separately for the GCT and the VTT data. A full description is found in Okunev (2004). An important goal of the data analyses is to achieve high polarimetric sensitivity of the order of $10^{-3} I_{\mathrm{c}}$, where $I_{\mathrm{c}}$ is the continuum intensity (near the Sun's limb). This is intricate because we need to retain high spatial resolution at the same time and it requires careful data handling.

\subsection{Data from the Gregory-Coudé Telescope}

\subsubsection{Observations: GCT}

The observations for the present work were obtained in June and August 2001. Several PFe were observed at high heliographic latitudes with $\left|\phi_{\odot}\right| \geq 60^{\circ}$ in the vicinity of the south and north poles. PFe were identified by means of the GCT's slit-jaw imaging in the continuum around $5820 \AA$. Spectropolarimetric data in the two Fe $\mathrm{I}$ lines $6301.5 \AA$ (Landé factor $g_{\text {eff }}=1.67$ ) and $6302.5 \AA(g=2.5)$ were obtained with the CzernyTurner spectrograph of the GCT. A Stokes $V$ polarimeter, consisting of a $\lambda / 4$ retarder plate and two crossed calcites, was mounted behind the entrance slit of the spectrograph. The slit width was 90-120 $\mu$ ( 0 . $\left.75-1^{\prime \prime}\right)$, the exposure times on a Thomson TH 7863 FT CCD chip with $384 \times 286$ pixels were in the range of 200-300 ms. To cover a two-dimensional field of view (FOV) we used a microscanner (Stolpe \& Kneer 1998), i.e. a device which moves the images across the slit. The chosen step width of the scanner corresponded to $0 .{ }^{\prime} 68$.

For the data analysis, dark frames and many flat field frames (with moving telescope pointing) were taken as well. The two beams of circularly polarized light at the exit of the $V$ polarimeter, $\frac{1}{2}(I+V)$ and $\frac{1}{2}(I-V)$, go through different optical paths in the spectrograph. To correct, during data analysis, for the resulting differential distortion of the two beams we took frames with a fixed pattern mounted in front of the entrance slit.

Instrumental polarimetric crosstalk $Q, U \rightarrow V$ may often be neglected since the Stokes $Q$ and $U$ parameters are themselves small. Yet the GCT possessed the feature that the small and only slowly varying instrumental polarization could be compensated by a properly rotated $\lambda / 2$ retarder plate in the coudé train (Sánchez Almeida et al. 1995). The polarization of the microscanner was compensated by a Bowen compensator (Koschinsky \& Kneer 1996)

\subsubsection{Data analysis: GCT}

Some of the data reduction follows standard recipes. Average dark frames were subtracted from the limb observations as well as from the flat fields. The spectrograms were rotated (with subpixel accuracy) to have the dispersion parallel to one axis of the CCD chip. The spectral lines were rectified to be oriented perpendicularly to the dispersion.

The gain tables were constructed separately for the $\frac{1}{2}(I+V)$ and $\frac{1}{2}(I-V)$ spectrograms. For this purpose, the averages of the many flat field spectrograms were collapsed to yield smooth spectra. The average flat fields were then divided by these spectra (expanded back again) which yielded the gain tables.

The spectrograms are contaminated by false light. This originates from infra-red light not fully suppressed by the order sorting pre-filter and from spectrographic scattered light mainly from the grating and from subsequent optics. The false light was accounted for by subtracting a constant light level, i.e. independent of wavelength, from the spectrograms such that the resulting average line profiles in the flat fields agree best with those from the Fourier Transform Spectrometer Atlas by Brault \& Neckel (quoted by Neckel 1999).

The distortions mentioned above are taken out by nonlinear stretching, to sub-pixel accuracy, of the $\frac{1}{2}(I+V)$ and $\frac{1}{2}(I-V)$ spectrograms with respect to each other. The stretching parameters are chosen such that data taken with the fixed pattern in front of the slit show minimum Stokes $V$ fluctuations in both direction, dispersion and perpendicular to it, i.e. after subtraction of the $\frac{1}{2}(I+V)$ and $\frac{1}{2}(I-V)$ spectrograms.

Noise filtering is an important part of the data analysis for achieving high polarimetric sensitivity. We chose the method of singular value decomposition (SVD, see e.g, Joliffe 1986). It is superior to low-pass filtering in the Fourier domain since it makes it possible to choose the number of retained singular values such that the rest does not exhibit any structure. Figure 1 shows in the leftmost image a Stokes $V$ spectrogram in the $6302 \AA$ range before filtering. The second image presents the same spectrogram after filtering where the 10 largest singular values are kept.

Obviously, the second image from the left in Fig. 1 shows systematic defects originating mainly from residual interference fringes, CCD imperfections and residual incomplete filtering. They were removed in the following way: we averaged many spectrograms which did not contain an obvious $V$ signal. The noise of the average was reduced again by an SVD retaining the first three highest singular values. The middle image of 
Table 1. FPI spectrometer settings during the VTT observations with dates, observed spectral line, exposure times, step widths of the spectrometer, its $F W H M$, the number of spectral positions in one scan, the number of frames taken at each wavelength positions, and the total spectral ranges around the lines.

\begin{tabular}{cccccccc}
\hline \hline Date & Spectral line & $\begin{array}{c}\text { Exposure } \\
\text { time }(\mathrm{ms})\end{array}$ & $\begin{array}{c}\text { Step width } \\
(\mathrm{m} \AA)\end{array}$ & $\begin{array}{c}F W H M \\
(\mathrm{~m} \AA)\end{array}$ & $\begin{array}{c}\text { No. spectral } \\
\text { positions }\end{array}$ & $\begin{array}{c}\text { No. Frames } \\
\text { per Spectr. Pos. }\end{array}$ & $\begin{array}{c}\text { Spectral range } \\
(\mathrm{m} \AA)\end{array}$ \\
\hline 15.06 .2001 & Fe II 6149.3 & 30 & 16.5 & 46.7 & 30 & 5 & 480 \\
28.04 .2002 & Fe I 6302.5 & 20 & 25 & 44 & 21 & 7 & 500 \\
29.04 .2002 & Fe I 6301.5 +6302.5 & 30 & 31.5 & 44 & 28 & 5 & $410+410$ \\
\hline
\end{tabular}
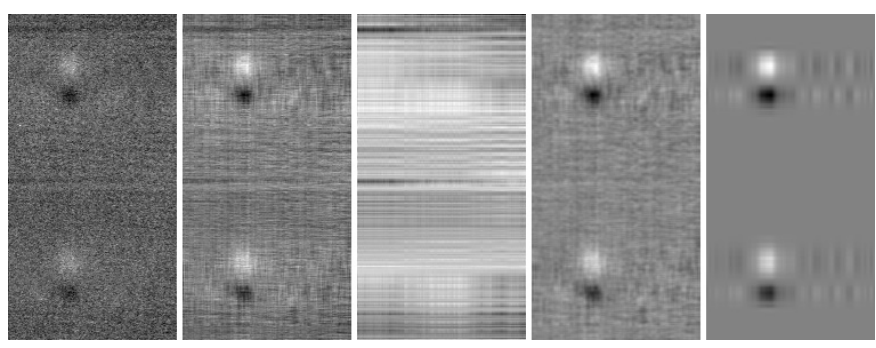

Fig. 1. Reduction of Stokes $V$ spectrograms comprising $1.67 \AA$ in the $6302 \AA$ range by means of SVD. The wavelength increases from bottom to top. The horizontal axis is the spatial direction along the slit corresponding to $28^{\prime \prime}$. From left to right: spectrogram after standard reduction before noise filtering; noise filtering with SVD retaining 10 out of 148 singular values; systematic artifacts from averages of many spectrograms containing, on average, no magnetic signal; $V$ spectrogram used for fitting (see text); after multiplication with binary mask and further SVD retaining one singular value.

Fig. 1 shows the defects which were then subtracted from the noise filtered spectrograms yielding the much cleaner $V$ spectrograms given in the fourth image of Fig. 1.

Finally, one can emphasize the very weak $V$ signals, well below the original noise level. For this a binary mask, with 0 in wavelength regions where no $V$ signal is expected and 1 otherwise, was multiplied with the $V$ spectrogram. Then an SVD was applied again, retaining only one (the highest) singular value. The result is shown in the rightmost panel of Fig. 1. This method was tested in two ways. First, an artificial $V$ spectrogram was superimposed with strong noise, much above the signal level. The highest value of SVD recovered correctly the positions of the original $V$ profiles. Then, only one of the two Fe I $V$ profiles was used for the SVD. The signal came out as in Fig. 1, but weaker. The use of both lines suppresses the noise further. In this way, a polarimetric sensitivity of 0.2 percent of the continuum intensity $I_{\mathrm{c}}$ (near the limb) could be achieved. Note that the resulting $V$ spectrograms are not considered as representing the correct $V$ signals. They will be used to obtain good starting values for fitting procedures (see Sect. 2.3 below).

\subsection{Speckle data from the Vacuum Tower Telescope}

\subsubsection{Observations: VTT}

With the VTT, spectropolarimetric observations of PFe were obtained in June 2001 and April 2002. The "Göttingen" two-dimensional spectrometer was used. It is based on wavelength scanning Fabry-Perot interferometers (FPIs, see Bendlin et al. 1992; Bendlin \& Volkmer 1995). The setup used for this investigation, which since several years has been working with two FPI etalons of high transmission, is described in Koschinsky et al. (2001, see Fig. 1 there). It also includes a Stokes $V$ polarimeter similar to the one used for the above GCT observations.

Several time series of spectral scans through the two Fe I lines at $6302 \AA$ and in addition through the Fe II $6149 \AA$ line were taken. Due to its specific Zeeman splitting, the latter line does not produce Stokes $Q$ and $U$ profiles and is thus insensitive to $Q, U \rightarrow V$ crosstalk. A spectral scan consists of positioning the spectrometer transmission at consecutive wavelength positions in the chosen spectral range. At each wavelength position, several short exposure images are taken. Table 1 summarizes the spectrometer settings during the observations. Strictly simultaneously with the spectrometric narrowband images we took broadband frames in the same wavelength region, with identical exposure times, and from the same area on the Sun. The short exposure times of 20-30 ms allow reconstruction of the two-dimensional images with speckle methods after the observations. The image scales on both broadband and narrowband CCD detectors corresponded to 0.11 per pixel.

For the data analysis, dark frames, flat field scans with moving pointing of the telescope, and scans with a fixed pattern in the primary focus of the telescope were also taken.

\subsubsection{Data analysis: VTT}

The analysis of images from FPI spectrometers is very complex. Details can be found in Hirzberger et al. (2001), Koschinsky et al. (2001), Janßen (2003), Janßen et al. (2003) and Okunev (2004). All frames were subject to dark correction, flat fielding and correction for image motion.

Broadband images. The broadband images from one scan form a burst consisting of 140-150 short-exposure frames. From these, one high-resolution image was reconstructed with the "Göttingen" speckle code (de Boer 1996, and references therein), which is widely in use, e.g. also ported to Utrecht (Rouppe van der Voort 2003). It uses the spectral ratio method by von der Lühe (1984) and the speckle masking by Weigelt (1977).

Two modifications to the standard speckle code were applied which improved the quality noticeably, that is the contrast of the reconstructed images: 1) we used many flat field scans, i.e. a low noise average flat field. 2) The low-contrast frames 
were rejected for the reconstruction; only the 100-120 best frames from a burst were admitted.

We refer to Fig. 6 for an example of the success of the reconstructions. The two uppermost panels show a subfield of the best image of a burst and the reconstructed image. The fine structure of the PF, having itself an extent of 1 .'0-1'.5, is clearly seen.

Narrowband images. The reconstruction of the images from the narrowband channel followed the method given by e.g., Keller \& von der Lühe (1992), Krieg et al. (1999) and Koschinsky et al. (2001). The estimate of the instantaneous optical transfer function (OTF) is obtained from the instantaneous images and the reconstructed broadband image. A leastsquares analysis yields the estimate of the narrowband image or, in Fourier space,

$$
O_{\mathrm{n}}(\boldsymbol{q})=H \cdot \frac{\sum I_{\mathrm{n}}(\boldsymbol{q}, t) I_{b}^{*}(\boldsymbol{q}, t)}{\sum\left|I_{b}(\boldsymbol{q}, t)\right|^{2}} \cdot O_{b}(\boldsymbol{q}) .
$$

Here, we have included an optimum filter $H$ after Brault \& White (1971), $I_{\mathrm{n}}$ are narrowband frames (i.e. Fourier transforms of subfields, cf. Krieg et al. 1999), and the summation runs over the images taken at one wavelength position. In some cases, the cut-off was kept at higher wavenumbers than prescribed by the optimum filter to preserve spatial resolution at the expense of retaining more noise.

The production of Stokes $V$ profiles from two-dimensional spectropolarimetric FPI data needs special care. We use the elaborate code developed in Göttingen and recently revised throughout by Janßen (2003), and we add two new features to it:

1. The mounting of the "Göttingen" FPI spectrometer in the collimated beam implies that the wavelength position of the FPI transmission peak varies over the FOV. The consequence is an intensity gradient over the $F O V$ especially in the flanks of line profiles. This variation of wavelength with respect to the position of the spectrometer step number is now corrected in the average flat field images before flat fielding, i.e. before determining the gain tables.

2. The above variation of the maximum FPI transmission over the $F O V$ is different for the two beams $\frac{1}{2}(I+V)$ and $\frac{1}{2}(I-V)$ denoted below by $I_{1}$ and $I_{\mathrm{r}}$, respectively. Thus, the correction has to be carried out very carefully. Yet, the line profiles in the two channels, after averaging over the whole FOV and over many scans, still showed differences of $\pm 2 \times$ $10^{-3} I_{\mathrm{c}}$. This results in an average $V$ profile with the same systematic offsets, though the average magnetic signal is expected to be negligible. We corrected for these systematic effects at each wavelength by a renormalization of the $I_{1}$ and $I_{\mathrm{r}}$ profiles according to

$I_{1}^{1}(x, y)=I_{1}^{0}(x, y) \cdot\left(\left\langle I_{1}^{0}\right\rangle+\left\langle I_{\mathrm{r}}^{0}\right\rangle\right) /\left(2\left\langle I_{1}^{0}\right\rangle\right)$ and

$I_{\mathrm{r}}^{1}(x, y)=I_{\mathrm{r}}^{0}(x, y) \cdot\left(\left\langle I_{1}^{0}\right\rangle+\left\langle I_{\mathrm{r}}^{0}\right\rangle\right) /\left(2\left\langle I_{\mathrm{r}}^{0}\right\rangle\right)$.

Here, the superscripts " 0 " and " 1 " denote the images before and after correction, respectively, and the averages are taken over the FOV and over many frames. This way, the systematic errors in the average $V$ profiles are completely removed.

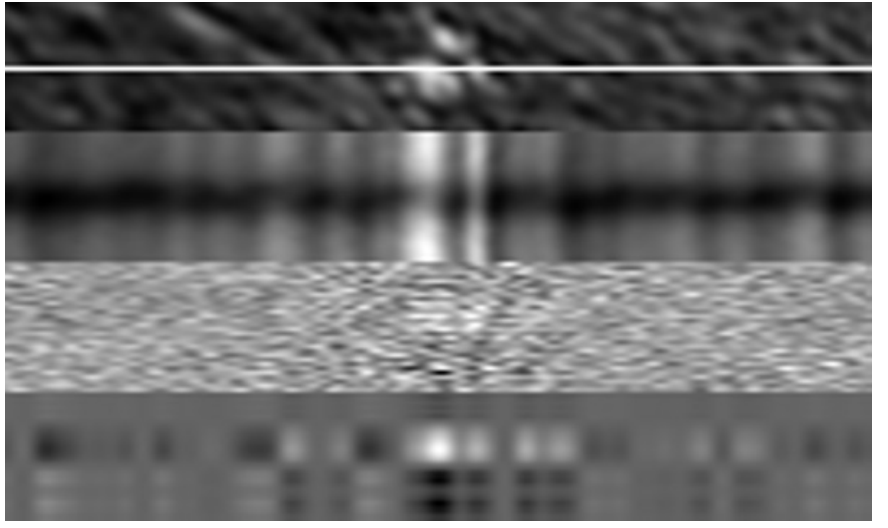

Fig. 2. SVD applied to an $x$ - $\lambda$ slice from an Fe II $6149 \AA$ A scan with the FPI polarimeter in the VTT. From top to bottom: subfield of speckle reconstructed image showing the virtual slit for the $x$ - $\lambda$ slice; Stokes $I(x, \lambda)$ spectrogram from the "slit"; original $V(x, \lambda)$ spectrogram from the "slit"; SVD analysis of $V$ spectrogram retaining the largest singular value. The sizes in the $x$ - and $\lambda$ direction are 26 " and $480 \mathrm{~m} \AA$, respectively.

\subsection{Signal recognition in polarimetric data}

We wish to detect low polarimetric signals, which may be buried in the noise, and yet want to retain high spatial and spectral resolution. For this, an image recognition algorithm was applied to the $V$ spectrograms. It minimizes the merit function

$\chi^{2}(\boldsymbol{a})=\sum_{i}\left(V_{\mathrm{obs}}\left(\lambda_{i}\right)-Y\left(\lambda_{i}, \boldsymbol{a}\right)\right)^{2}$.

The analytic function $Y(\lambda, \boldsymbol{a})$ representing the $V$ profiles is chosen as a linear combination of two Gaussians and a parabola. It contains in the vector $\boldsymbol{a}$ nine free parameters according to

$$
\begin{aligned}
Y(\lambda, \boldsymbol{a})= & a_{0} \mathrm{e}^{-\left(\lambda-a_{1}\right)^{2} /\left(2 a_{2}^{2}\right)}+a_{3} \mathrm{e}^{-\left(\lambda-a_{4}\right)^{2} /\left(2 a_{5}^{2}\right)} \\
& +a_{6}+a_{7} \lambda+a_{8} \lambda^{2} .
\end{aligned}
$$

For properly corrected data only the linear term or even just the constant offset is needed for fast convergence of the fitting algorithm.

Obviously, the double Gaussian cannot retrieve $V$ profiles with three lobes appearing in observations with mixed polarity within the resolution element (see the examples in Sánchez Almeida \& Lites 2000). Yet they are efficient in detecting weak signals, $<1 \%$ of $I_{\mathrm{c}}$, in the $F O V$, in estimating $V$ amplitudes, the separation of $V$ lobes, and the $V$ zero-crossings for velocity determinations.

For a convergence of the fitting algorithm a good choice of the initial parameter set $\boldsymbol{a}$ is important. It is obtained from an SVD described above retaining only the highest singular value. This yields good guesses of the amplitudes and the positions of the $V$ profiles as seen in Fig. 1 and demonstrated once more in Fig. 2. There, SVD was applied to $x$ - $\lambda$ slices, i.e. to $V$ images with coordinates in $x$ - and wavelength direction.

Figure 3 shows, from VTT data, on the left a reconstructed broadband image of a PF and surrounding area overlaid with contours where magnetic signatures have been detected with the above SVD and subsequent profile fitting. The magnetic patches possess sub-arcsecond structure. Remarkably, the 

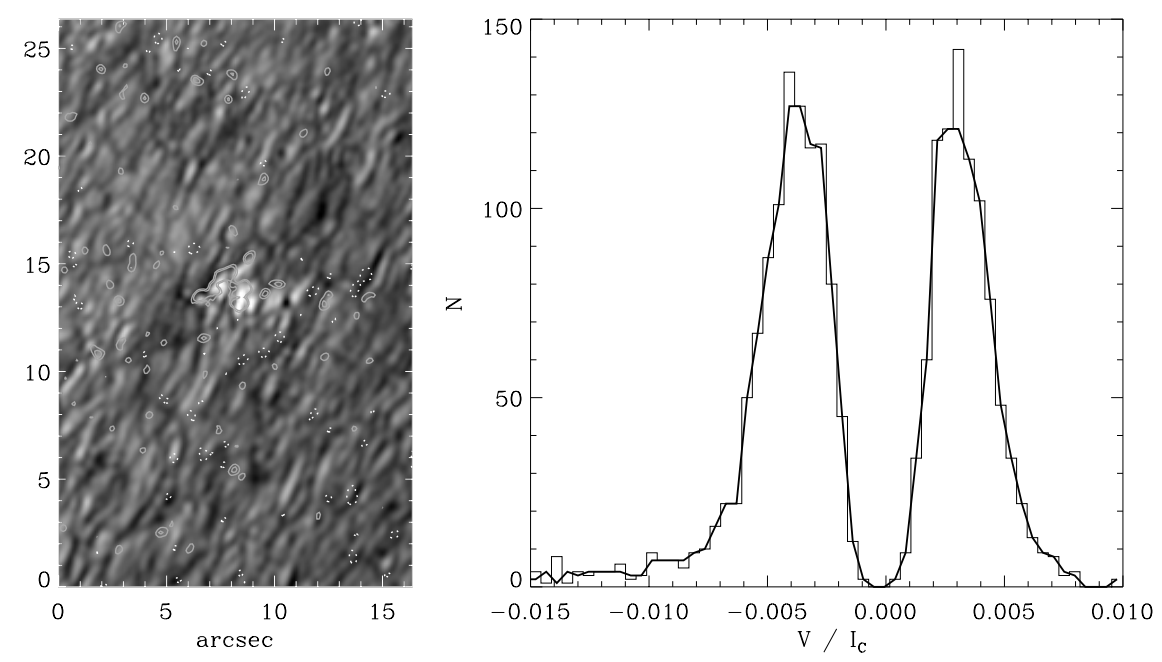

Fig. 3. Left image: speckle reconstructed broadband image of polar facula with surrounding area overlaid with contours of detected magnetic fields; right panel: distribution of extrema of the red minus blue $V / I_{\mathrm{c}}$ lobes from the same $F O V$.

strongest magnetic signals occur displaced from the smallscale brightenings of the PF towards disk center by approximately 0.5 . Moreover, some of the PF brightenings appear only little related to magnetic features, the latter appear more in the dark lanes between the brightenings. We note also that many magnetic features occur outside PFe showing no preferential polarity. This is seen in the right panel of Fig. 3 where the distribution of the amplitudes of the red minus the blue lobe of the $V$ profiles is drawn. Around $\left|V_{\text {extr }}\right| / I_{\mathrm{c}} \approx 0.004$ both polarities occur equally often. The tail at $V / I_{\mathrm{c}}<-0.008$ reflects the strong amplitudes from the PF magnetic fields. The gap around $V=0$ results presumably from the observational bias since a definite recognition for $|V| / I_{\mathrm{c}}<0.004$ in pixels of $0 .{ }^{\prime} 1$ size becomes increasingly difficult with decreasing amplitude. That is, with decreasing SNR the signal detection algorithm rejects more and more profiles.

A test of the reliability of the magnetic signatures and a further suppression of noise was done in the following way. The $V$ signals found were grouped into bins of different ranges of (signed) amplitude. The measured, not fitted, profiles in each bin were then averaged. Figure 4 gives some examples of the resulting profiles together with their fits from Eqs. (3) and (4). The noise depends on the number of contributing measurements. If the detections in the single profiles were incidental the averaging would essentially leave noise. It is, however, seen that the original noise (of approximately $1 \%$ of $I_{\mathrm{c}}$ ) is strongly reduced and $V$ profiles with low amplitude (of 0.001-0.002 $I_{\mathrm{c}}$ ) emerge clearly. We recall again that the pixel size corresponds to $0 . ' 1$.

\section{Results}

\subsection{Photometric properties}

The brightness represents an observational constraint for modelling the structure of PFe. We relate the intensities of the observed PFe to the average intensity of their ambient area. For this purpose, a second order curve was fitted to the quiet Sun continuum intensities of the spectrograms from the GCT, or, in the case of the FPI observations from the VTT, a second order
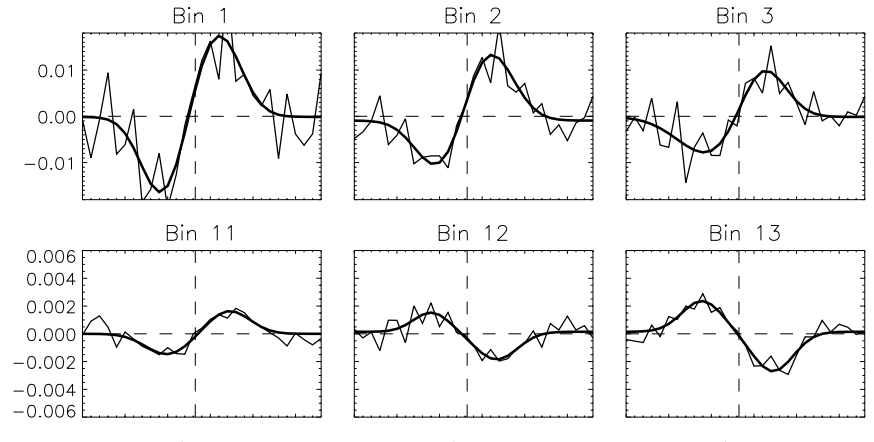

Bin 13
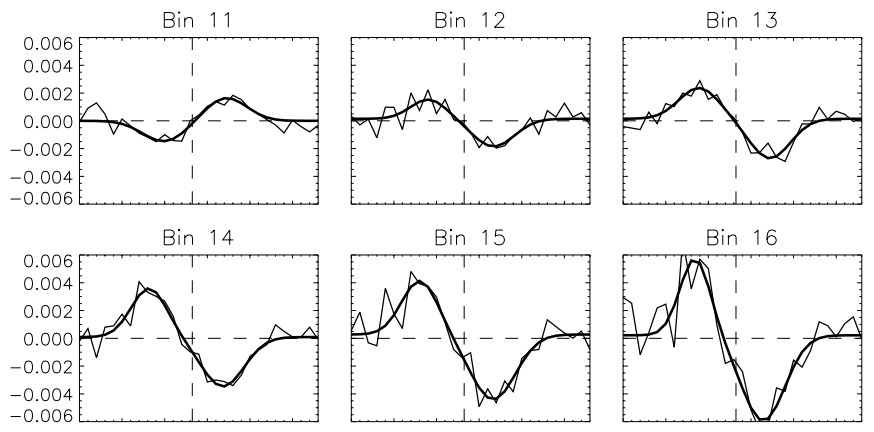

Fig. 4. Examples of averages (from amplitude bins) of observed $V$ profiles in Fe II $6149 \AA$ (thin curves) and fitted $V$ profiles (thick curves) near polar faculae. The abscissae are in scanning steps of the spectrometer, the ordinates in $V / I_{\mathrm{c}}$.

surface to the broadband images. The contrast $C$ is then defined in the usual way by

$C=\frac{I_{\mathrm{PF}}-\bar{I}_{\mathrm{QS}}}{\bar{I}_{\mathrm{QS}}} \times 100$,

where $I_{\mathrm{PF}}$ and $\bar{I}_{\mathrm{QS}}$ denote the PF intensity and the local quiet Sun intensity of the second order curve (or surface), respectively. In Eq. (5) we have applied a factor of 100 to present $C$ in percent $(\%)$.

Figure 5 shows the contrasts obtained in the present work from polar facular points at various heliocentric positions, with $\mu=\cos \theta$. One notices the following photometric properties:

1. The contrasts of PF points decreases from $C \approx 50 \%$ at $\mu=$ 0.37 towards the limb to $C=15-20 \%$ at $\mu=0.15$.

2. The contrasts in the not reconstructed VTT images and in the spectrometric GCT data are similar. They are approximately a factor of two lower than after the image 


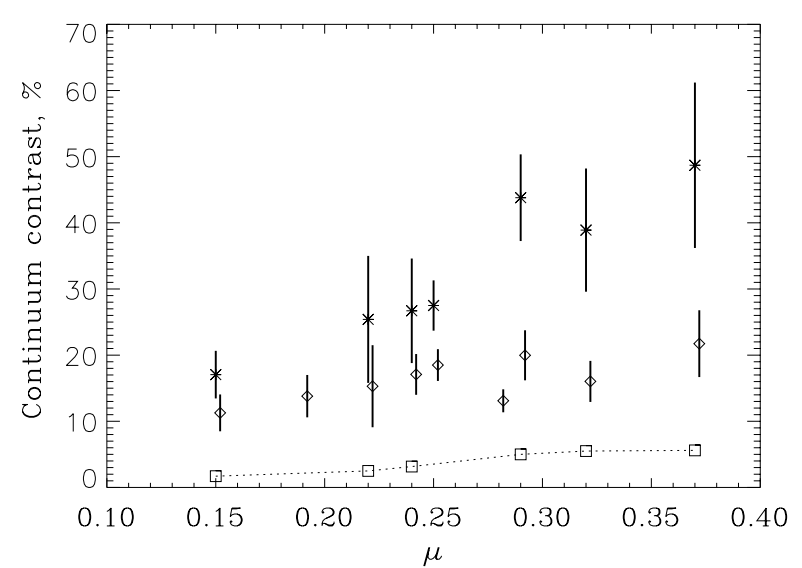

Fig. 5. Part of center-to-limb variation of contrasts of polar facular points. $* * *$ : from speckle reconstructed broadband images (VTT); $\diamond \diamond \diamond$ : from original broadband images (VTT) and continuum contrasts from spectrograms (GCT); $\square$ व: granular rms contrast in reconstructed broadband images (VTT). The vertical bars indicate $1 \sigma$ variation of the measurements.

reconstruction. The increase of the contrast by reconstruction is somewhat higher for brighter structures at larger $\mu$.

3. A maximum of the contrast of PF points is not seen, it occurs at $\mu \geq 0.4$.

Hitherto, contrast observations of PFe with high spatial resolution were not yet available. Contrasts of faculae, which are mainly located in the active region belt, have been measured by Lawrence \& Chapman (1988) with a spatial resolution of approximately $2^{\prime \prime}$ at $\lambda=6264 \AA$. They obtain contrasts in the range of $3-8 \%$ for $\mu<0.5$. Auffret \& Muller (1991), from high-resolution (0'.23) photographic observations at $\lambda=5750 \AA$ near the Sun's equator, presented the centerto-limb variation $(C L V)$ of network bright points with a maximum contrast of up to $40 \%$ at $\mu \approx 0.4$. These authors note that the most significant $C L V$ is obtained only for bright points of medium size of 0!'4-0!'7. Ortiz et al. (2002) analysed MDI data at $\lambda=6768 \AA$ with moderate spatial resolution. For strong magnetic fields they find contrasts of up to $6 \%$ with a maximum indicated near $\mu=0.3$. Sánchez Cuberes et al. (2002, see also references therein) measured contrasts with the Swedish Vacuum Solar Telescope on La Palma at wavelengths of $0.8 \mu \mathrm{m}$ and $1.55 \mu \mathrm{m}$ and obtain for the former wavelength a maximum contrast of $4-8 \%$ at $\mu=0.3$.

The contrast amplitudes found here for PFe, cf. Fig. 5, are in good agreement with the results of Auffret \& Muller (1991) for network bright points. (The $\mu$ position of the maximum of the contrast of PFe could not be measured from Fig. 5.) The two data sets have similar spatial resolution. Yet we note that in the present work the high contrasts occur at smaller sizes than the values of 0.'4-0.'7 given by Auffret \& Muller (cf. Sect. 3.2 below). The results here are also compatible with the findings of other authors (Lawrence \& Chapman 1988; Ortiz et al. 2002; Sánchez Cuberes et al. 2002), considering the limited spatial resolution of these studies. For instance, an image reconstruction of the Sánchez Cuberes et al. data would likely increase the contrasts by a factor of two, while the difference of the source functions at the different wavelengths (6300 $\AA$ vs. $8000 \AA)$
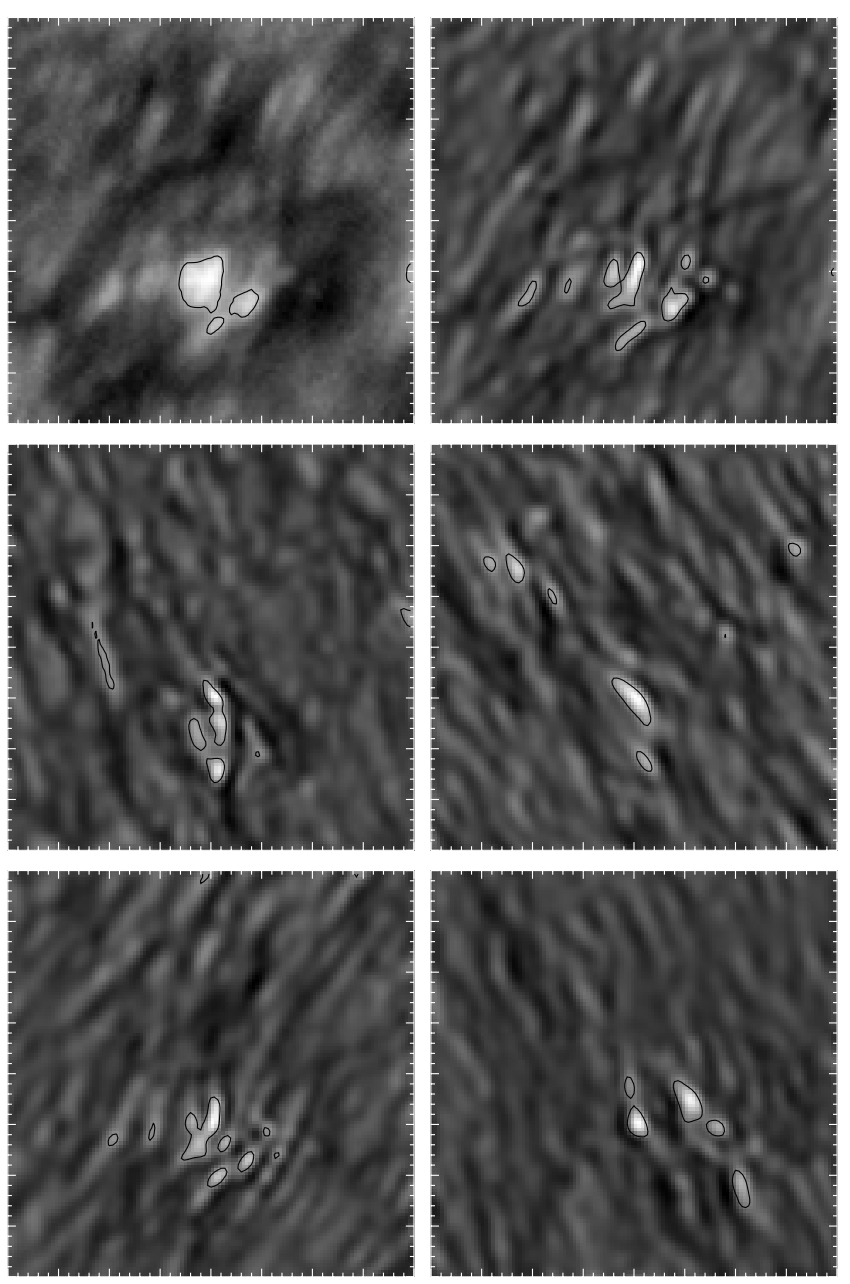

Fig. 6. $8^{\prime \prime} \times 8^{\prime \prime}$ broadband images (from VTT) as examples of PF fine structure. The uppermost two panels show the non-reconstructed (left) and reconstructed (right) image from the same FOV. The black contours enclose areas with brightness enhancement $>3 \sigma$ of the granulation contrast.

and the different heights of formation of the continuum intensities at these wavelengths can account for another factor of 2-3 (Pérez Rodríguez \& Kneer 2002).

\subsection{Fine structure and size}

At medium spatial resolution, $\mathrm{PFe}$ appear as diffuse patches with sizes of $1^{\prime \prime}$ or larger. At high spatial resolution, PFe are broken up into short chains of pearl-like structures. Figure 6 gives a few examples, where the uppermost two images are the not reconstructed (left) and the reconstructed image (right) from the same $F O V$ of $8^{\prime \prime} \times 8^{\prime \prime}$.

The average distance of the "pearls" along regular chains of approximately 0.'5 may serve for the modelling of PFe and of small-scale MFTs as their building blocks. The extent of the small brightenings in the reconstructed images perpendicular to the limb is often as small as the diffraction limit of the VTT of approximately 0 ' 2 . This together with the notion that some of the fine structures appear with regular, almost roundish shape 
of the same extent, 0 '.2, leads to the conclusion that we have still not yet fully resolved the substructure of PFe.

\subsection{Temporal evolution}

On June 15, 2001, two FPI-VTT time series were obtained from $\mathrm{PFe}$ close to the south pole of the Sun under good and stable seeing conditions. The first series consists of 20 scans at a cadence of $49 \mathrm{~s}$, the second of 40 scans (at the same cadence) with a gap of four minutes in the middle. These data were reduced completely and two movies of broadband images were produced, which are available to the interested ${ }^{1}$. For the latter purpose, a few further reduction processes were applied:

1. Global shifts between neighboring images were corrected for by cross-correlation techniques.

2. To eliminate (solar) brightness oscillations and brightness flickering from one image to the next due to (necessarily) incomplete image restoration, a conical low-pass filter was applied to the Fourier transform of the $(x, y, t)$ data cubes.

3. The gap of $4 \mathrm{~min}$ in the second time sequence was filled by linear interpolation.

Figure 7 presents the first of the two time sequences of $16 \mathrm{~min}$ duration. The temporal behaviour of PFe is very different from that of the granular convection. At low resolution, one does not see much difference of the PF in the first and in the last image. Yet at high resolution, $\mathrm{PFe}$ evolve on short time scales, sometimes noticeably between subsequent images with 49 s time difference. Thus, $\mathrm{PFe}$ are highly dynamical, active phenomena. Their constituents, the tiny, bright structures, are permanently evolving, they move, merge and split, decay and flare up again.

Such a behaviour probably reflects the interaction between the convective motions and individual MFTs. Horizontal flows can shake, bend and displace individual flux tubes. Moreover, magnetic tubes can be squeezed by the varying external pressure which will change the magnetic field strength and, accordingly, will affect the optical thickness of the flux tubes. All this can change the contribution of the facular atmosphere to the emergent intensity and in this way can change the intensity pattern.

A similar behaviour is expected for network magnetic fields. They are formed, as is commonly accepted, by horizontal flows, which sweep and concentrate the magnetic fields towards the network boundaries. This picture is in agreement with numerical simulations (e.g., Emonet \& Cattaneo 2001; Vögler \& Schüssler 2003). Yet PFe belong to another class of solar magnetic activity. They take part in the global magnetic cycle of the Sun and probably emerge from deep down as already organized bundles of MFTs. So, such a highly dynamical behaviour of the PF substructure should not be associated with the formation of faculae but with their evolution due to the interplay between convection and the global magnetic field.

The dynamic behaviour of PFe may be relevant for the transfer of energy to the corona. Choudhuri et al. (1993) suggested kink waves for coronal heating (see also Wellstein et al. 1998). According to Marsch \& Tu (1997) and Marsch (1998)

\footnotetext{
${ }^{1}$ http://www.uni-sw.gwdg.de/ olok/pf
}
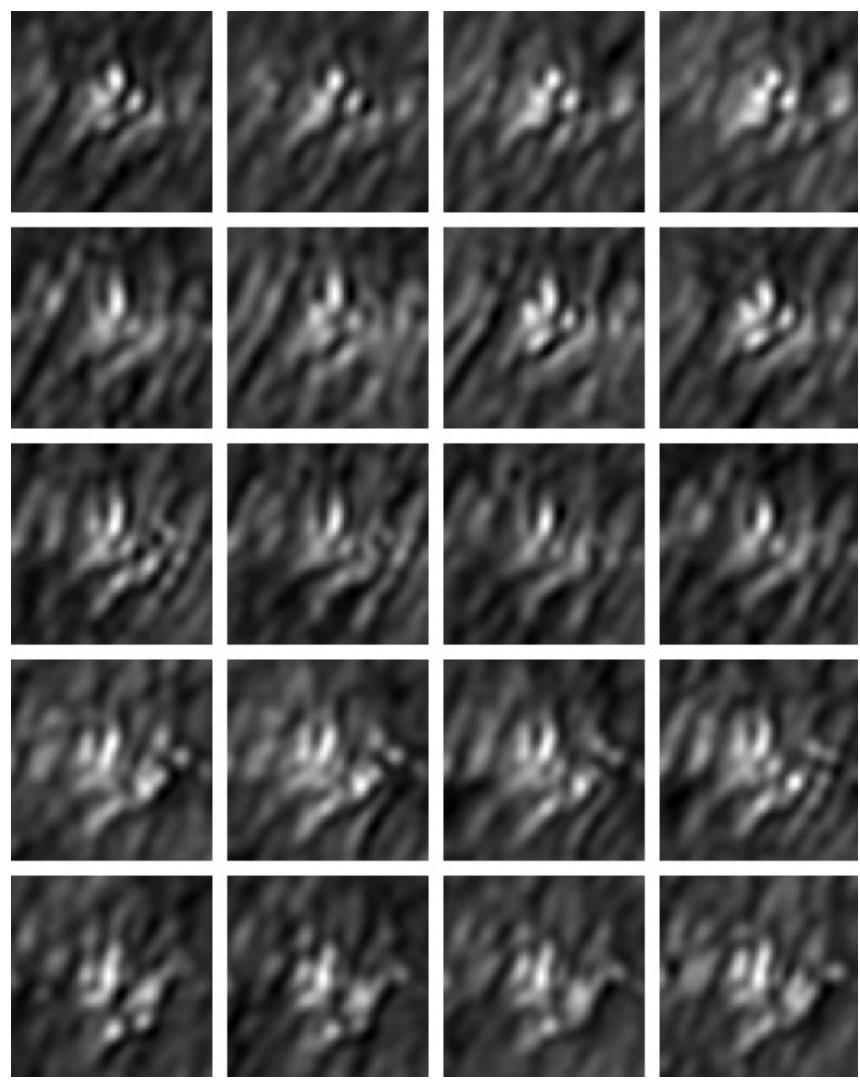

Fig. 7. Temporal evolution of a polar facula within $16 \mathrm{~min}$. The sequence runs row by row and from left to right, starting with the uppermost left image. The $F O V$ is $4^{\prime \prime} \times 4^{\prime \prime}$.

coronal heating and wind acceleration are achieved by highfrequency $(>1 \mathrm{~Hz})$ Alfvén waves and cyclotron resonances in open magnetic funnels. Such short-period events are yet undetectable with the temporal resolution limits set by today's technology.

\subsection{Spectropolarimetric properties}

Further information on the structure of PFe comes from spectropolarimetry. Line profiles and polarimetric signals may be used to model the temperature and magnetic field structure and to draw conclusions on velocities. Yet as shown above, from the high spatial resolution appearance of $\mathrm{PFe}$ one must expect inhomogeneities along the line-of-sight ( $L O S$ ) which makes the interpretation of spectropolarimetric data a difficult task.

Figure 8 gives an example of Stokes $I$ and $V$ profiles around $\lambda=6302 \AA$ from the GCT observations. The PF intensities are higher than the profiles from the surrounding area throughout the line profiles. When normalized to the local continuum, the $I$ profiles from $\mathrm{PFe}$ and quiet Sun appear very similar, the $\mathrm{PF}$ profiles being somewhat broader and less depressed near the line center.

FPI-VTT spectropolarimetric data are depicted in Fig. 9. The spectral resolution of these data is lower than that of the GCT data, while their spatial resolution is better, 0.5 or better compared to approximately 1 .'0 for GCT spectroscopy. We can recognize the same behaviour of the Fe r $6302.5 \AA$ line as in the 

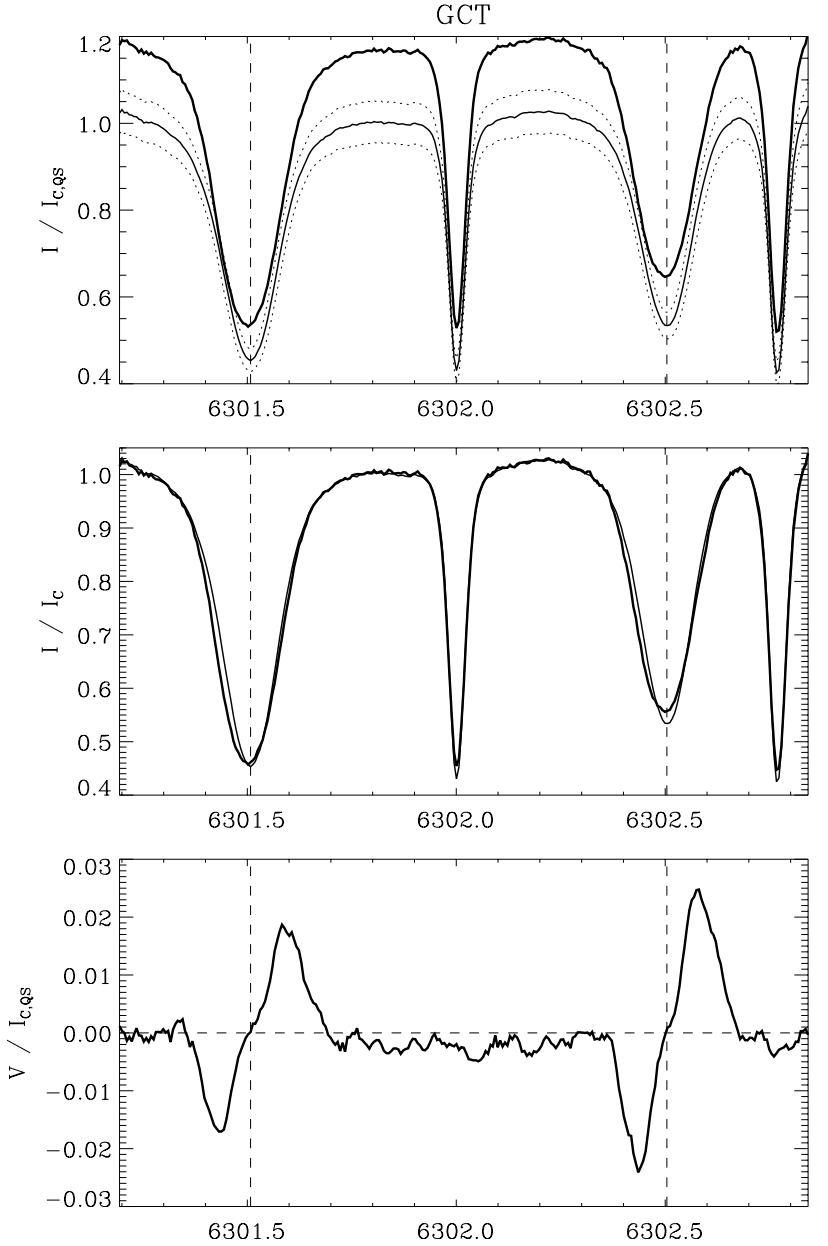

Fig. 8. Spectrograms from a polar facula (solid thick profiles) and ambient atmosphere (thin profiles) around $6302 \AA$ from GCT. Upper panel: Stokes $I$ profiles normalized to the "continuum" (local maximum at $6301.8 \AA$ ) of the profile from the ambient atmosphere. The dotted profiles indicate the rms fluctuation in the non-facular atmosphere. Middle panel: I profiles normalized to the local "continuum"; lower panel: Stokes $V$ profiles from the polar facula normalized to the "continuum" of the ambient photosphere.

GCT data. In contrast to this Fe I line, the Fe II $6149.2 \AA$ line is significantly stronger, broader and more depressed in PFe than in the ambient area.

\subsection{Magnetic fields}

Homann et al. (1997) have shown that the Stokes $V$ signals from $\mathrm{PFe}$ require kilo-Gauss fields. We arrive at the same field strengths from the modelling to be described in a subsequent publication (see also Okunev 2004). Here, we restrict ourselves to the weak field approximation $(W F A)$ and perform, beyond that, a preliminary calibration to estimate the magnetic field strengths in the present observations of polar facular points. In the $W F A$ the $V$ profile is given by (cf. Landi Degl'Innocenti 1992)

$V(\lambda)=-4.67 \times 10^{-5} \bar{g} \lambda_{0}^{2} B_{\mathrm{eff}} \frac{\mathrm{d} I_{\mathrm{m}}(\lambda)}{\mathrm{d} \lambda}$,
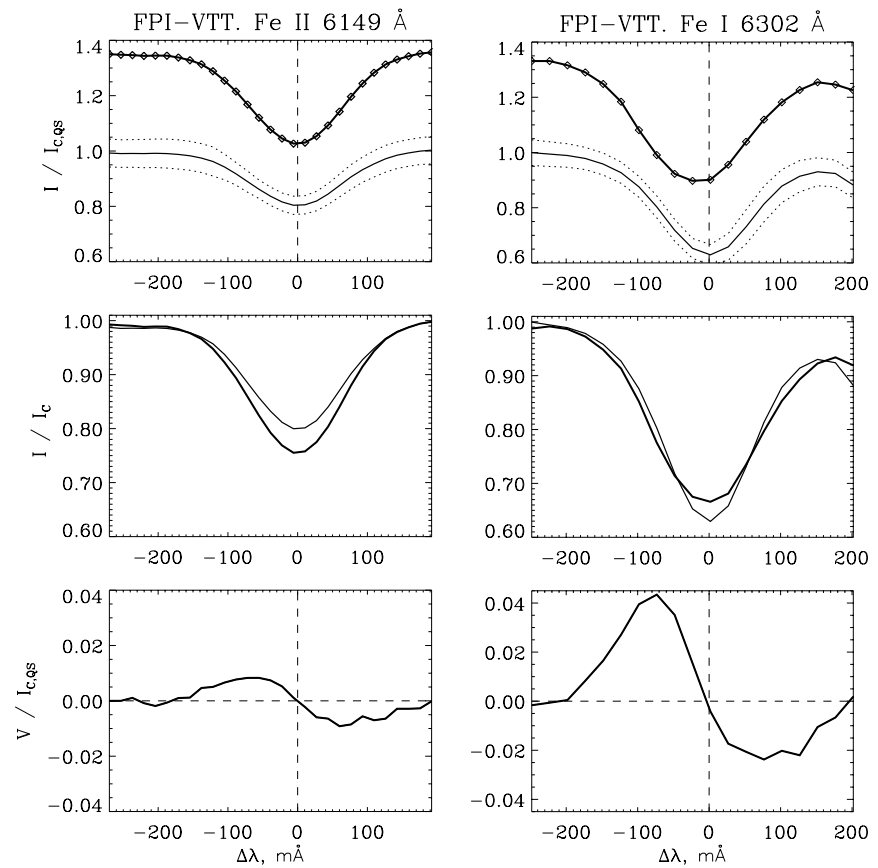

Fig. 9. Stokes profiles from a polar facula (thick profiles) and ambient atmosphere (thin lines) of the Fe II $6149 \AA$ and Fe I $6302 \AA$ lines from FPI-VTT data. Upper panel: Stokes $I$ profiles normalized to the maxima of the profiles from the ambient atmosphere. The dotted profiles indicate the rms fluctuation in the non-facular atmosphere. Middle panel: I profiles normalized to the local maximum; lower panel: Stokes $V$ profiles from the polar facula normalized to the "continuum" of the ambient photosphere.

Table 2. Magnetic field strengths (in G) estimated from the WFA.

\begin{tabular}{cccc}
\hline \hline Spectral Line & Telescope & $\cos \theta$ & $B_{\text {eff }}$ \\
\hline 6149.3 & VTT & $\sim 0.3$ & $\sim 250-300$ \\
6301.5 & GCT & 0.2 & $\sim 100$ \\
6302.5 & GCT & 0.2 & $\sim 80$ \\
\hline
\end{tabular}

where $B_{\text {eff }}$ is measured in $\mathrm{G}$ and $\lambda$ in $\mathrm{cm}, \bar{g}$ is the effective Landé factor and $I_{\mathrm{m}}(\lambda)$ is the (magnetically non-affected) intensity profile in the magnetic structure. The effective magnetic field strength is given by

$B_{\text {eff }}=f \cos \gamma B$,

with $f$ the filling factor and $\gamma$ the angle between the magnetic field and the $L O S$.

The values of the $V$ extrema relative to the continuum intensity of the ambient quiet Sun, measured in PFe, are in the range of $\left|V_{\text {extr }}\right| / I_{\mathrm{c}, \mathrm{QS}}=0.01-0.06$. The individual values depend on the heliocentric position, on the spatial resolution, and on the spectral line. The derivative of the intensity in Eq. (6) was calculated numerically from the observed $I$ profiles. Some caution is advised also here, since the observations are spatially not fully resolved and contain contributions from non-magnetic regions. Table 2 gives some typical determinations of $B_{\text {eff. }}$ The ratio of $B_{\mathrm{eff}, 6301.5} / B_{\mathrm{eff}, 6302.5}=1.25$ from the GCT observations is the same as the one found by Domínguez Cerdeña et al. (2003) in quiet Sun intra-network regions. FPI-VTT high 

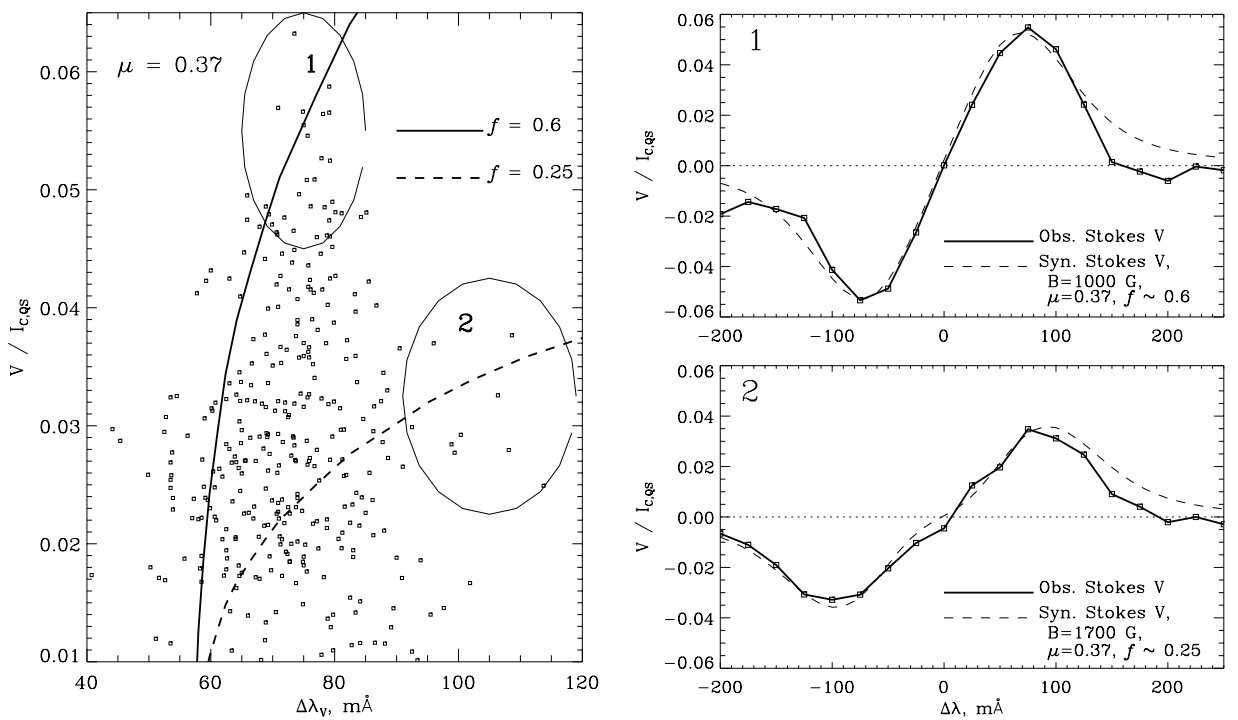

Fig. 10. Left panel: observed (open squares) and simulated $V$ amplitudes vs. separation of the $V$ extrema in Fe i 6302.5. Right panels: two examples of $6302.5 \mathrm{~V}$. The observations in the upper panel are averages from group 1 (encircled in the left panel), those from the lower panel are averages from group 2 . The simulations are performed with the VAL C model (see the text). spatial resolution results from $\mathrm{Fe}_{\mathrm{I}} 6302.5 \AA$ will be discussed below.

The other extreme case occurs when the magnetic field is so strong that the separation of the Stokes $V$ extrema is proportional to the field strength

$\Delta \lambda_{V, \text { extr }}=2 \times \Delta \lambda_{B}=2 \times 4.67 \times 10^{-5} \bar{g} \lambda_{0}^{2} B$.

For Fe I 6302.5 this happens at field strengths $\geq 1500 \mathrm{G}$, for the other observed lines at still stronger fields.

We will not present results of determinations of $B$ under the assumption of this strong field regime. Instead, we perform a simple calibration using the VAL C model atmosphere of the quiet Sun (Vernazza et al. 1981). Vertical constant magnetic fields of various strengths are assumed. The $V$ profiles emerging at an angle $\theta$ between the magnetic field and the LOS are calculated and a filling factor $f$ is applied. Finally, to account for the limited spectral resolution of the FPI-VTT spectrometer, the $V$ profiles are convolved with the FPI's Airy function.

Figure 10 compares PF observations in Fe I 6302.5 at $\mu \approx$ 0.37 with such simulations. The left panel gives the $V$ amplitudes vs. their separation. It shows that large $V$ amplitudes of $\left|V_{\text {extr }}\right| / I_{\mathrm{c}, \mathrm{QS}} \approx 0.06$ have been measured, thanks to the high spatial resolution. Accounting for the depression of the $V$ profiles by the spectrometer's broadening function, which from our experience amounts to a factor of approximately 1.5 , and considering the expected decrease of $|V|$ when observing close to the solar limb, such large amplitudes require both strong fields and large filling factors $f$. The two right panels of Fig. 10 give examples of observed $V$ profiles, as averages of group 1 (of the left panel) and of group 2. The synthetic profiles, which fit well the observed characteristics, are obtained from kilo-Gauss fields. Further evidence for kilo-Gauss fields comes from the ratio of 1.25 of the effective field strengths $B_{\text {eff }}$ measured simultaneously in the 6301.5 and $6302.5 \AA$ lines which, according to Domínguez Cerdeña et al. (2003), also requires strong fields.

A word on the magnetic polarity of $\mathrm{PFe}$ is appropriate. Although the surroundings of the PFe exhibit small-scale magnetic fields of both polarities (cf. Fig. 3) as in the quiet Sun network, the observed PFe proper almost always had the magnetic polarity according to the temporal position along the solar cycle, which, in 2001 and 2002, was shortly after the reversal of the general polar magnetic field. Four PFe at the solar north pole showed negative polarity, seven at the solar south pole had positive polarity. In one observation, right at the magnetic field reversal in December 2000, two PFe at the same pole with opposite magnetic polarity were detected. These findings, admittedly with low statistics, confirm the suggestion by Homann et al. (1997) that the magnetic polarity of PFe agrees with that of the general solar magnetic field ${ }^{2}$. It thus appears unlikely that the coronal plumes originate from bipolar PF regions, as one might be tempted to assume from the study of the polar corona by Wilhelm et al. (1998).

\subsection{Velocities}

The $L O S$ velocities in PFe were determined from their corresponding $I$ profiles and from the zero-crossings of their $V$ profiles. The $I$ profiles were smoothed with a three-point boxcar. Then the line cores were fitted by a fourth order polynomial from which the Doppler shift was determined. The zerocrossings are measured from the Gaussians fitted to the $V$ profiles according to Eqs. (3) and (4). Zero velocity was defined as the average velocity over the $F O V$, which was found to agree within $\pm 32 \mathrm{~m} \mathrm{~s}^{-1}$ with the velocity of the average line profile. Following Schröter (1956, Eq. (37) and Figs. 8 and 9 there for $\mu \approx 0.37$ ), this reference zero velocity contains actually, for medium strong lines, a residual, granular blueshift corresponding to approximately $-0.24 \mathrm{~km} \mathrm{~s}^{-1}$, relative to rest on the solar surface.

Figure 11 gives an example of the results from VTT-FPI observations of Fe I 6302.5 at $\mu \approx 0.37$. The velocities vs. the

\footnotetext{
${ }^{2}$ For a magnetographic "butterfly diagram" see http://science.nasa.gov/ssl/pad/solar/images/ magbfly.gif and http://science. nasa.gov/ssl/pad/solar/dynamo.htm
} 


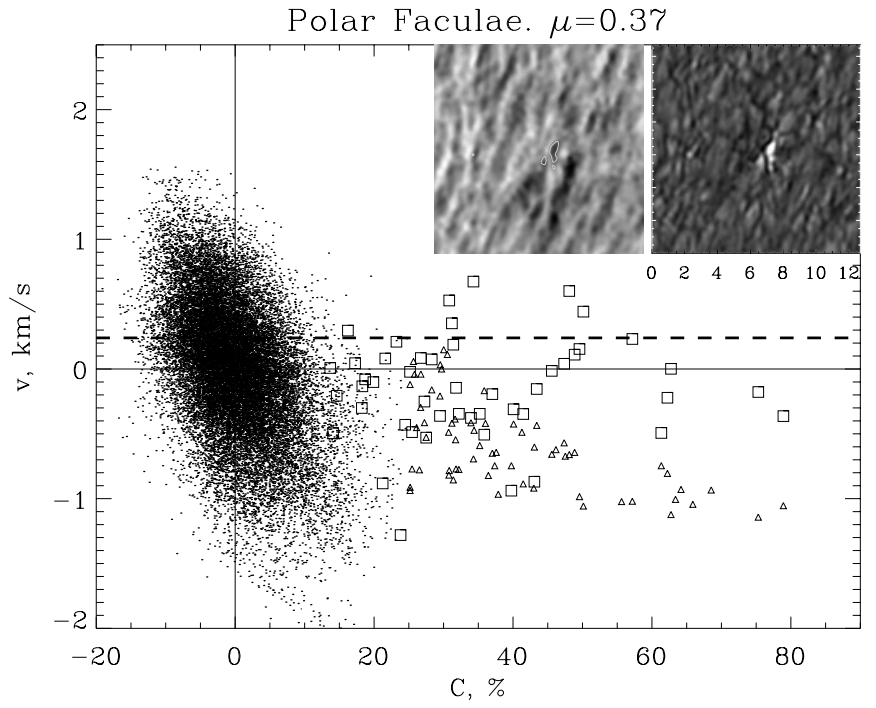

Fig. 11. $L O S$ velocities measured in $\mathrm{Fe}_{\mathrm{I}} 6302.5$ at $\mu \approx 0.37$ vs. intensity contrast. The dots stem from the non-magnetic region within the $F O V$, the squares from the zero-crossings of the $V$ profiles of the facular points, and the triangles from their $I$ profiles. Negative velocities are towards the observer. The horizontal dashed line at $+0.24 \mathrm{~km} \mathrm{~s}^{-1}$ gives the absolute zero velocity, rest at the solar surface, according to Schröter (1956). The inlay shows the Dopplergram and the reconstructed broadband image, with scale in arcsec.

intensity contrasts are depicted (negative velocities are towards the observer).

The velocities from the non-magnetic granular structures behave as expected, even at $\mu \approx 0.37$. Convective motions are towards (away from) the observer for bright (dark) structures.

The squares in Fig. 11 represent the velocities measured from the $V$ zero-crossings, where in the present example the locations of strong $V$ signals coincide with those of high continuum intensities. The scatter of these velocities is large, $\pm 1 \mathrm{~km} \mathrm{~s}^{-1}$. This is not due to noise, i.e. random measurement errors, which were estimated to be less than $0.25 \mathrm{~km} \mathrm{~s}^{-1}$. It is inherent to the properties of single flux tubes and reflects their dynamic behaviour.

The average velocity of the gas within the flux tubes is $-(0.15 \pm 0.06) \mathrm{km} \mathrm{s}^{-1}$. The velocity relative to rest on the solar surface then amounts to $-0.39 \mathrm{~km} \mathrm{~s}^{-1}$. This value is likely to be even more negative, towards the observer, since the zero-crossings tend to be biased towards redshifts on the following ground: Most $V$ profiles exhibit stronger blue than red lobes. The convolution with the FPI spectrometer's Airy function then yields, according to numerical tests, a redshift of the $V$ zero-crossings corresponding to $0.2-0.5 \mathrm{~km} \mathrm{~s}^{-1}$.

The high velocities of $0.5-1.0 \mathrm{~km} \mathrm{~s}^{-1}$ towards the observer from the $I$ profiles (triangles in Fig. 11) are surprising. We note that such values are found in all our high spatial resolution observations. They are different from the velocities determined from the $V$ zero-crossings and thus refer mainly to bright gas surrounding the magnetic fields. This is difficult to comprehend, a priori, in view of numerical MFT simulations where it is found that small-scale flux tubes are embedded in regions of downflowing cool gas (Deinzer et al. 1984a,b; Vögler \& Schüssler 2003). To understand the $I$ and $V$ profiles including the deduced velocities, from PF flux tubes and their surroundings, radiative transfer modelling of these dynamic features observed near the limb are needed.

\section{Conclusions}

This study dealt with polar faculae (PFe) of the Sun, which constitute an important part of the magnetic solar cycle. Since they belong to the global solar magnetism they are very likely phenomena deeply rooted in the Sun's interior.

Here, we concentrated on the fine structure and the dynamics of PFe in order to reveal their rôle in the magnetic activity at the poles of the Sun. For this purpose, we aimed at obtaining measurements in Stokes $I$ and $V$ of high spatial and spectroscopic resolution with the Gregory-Coudé Telescope and the Vacuum Tower Telescope at the Observatorio del Teide. The careful application of special methods of observation and data analysis, image speckle reconstruction and singular value decomposition, lead to the detection of a wealth of substructure and dynamic phenomena of PFe. We summarize our main results as follows:

1. A polarimetric sensitivity of $|V| \approx 2 \times 10^{-3} I_{\mathrm{c}}$ at a spatial resolution of 0.5 was achieved. This revealed many smallscale, weak magnetic flux areas of both magnetic polarities surrounding the PFe proper, like in the quiet Sun near disk center (e.g. Domínguez Cerdeña et al. 2003).

2. PFe exhibit a high contrast of $C \approx 0.5$ at $\mu=\cos \theta \approx 0.4$, which monotonically decreases still further to the limb, i.e. a maximum of $C$ at $\mu<0.4$ is not seen.

3. PFe possess fine structure in both brightness and magnetic fields down to the spatial resolution limit $(0.25$ for speckle reconstructed broadband images and 0.5 for magnetograms). The broadband images from facular points appear somewhat displaced, $\sim 0$ '. 5 , towards the limb with respect to the magnetic features.

4. Polar facular points are highly dynamic, they change noticeably their brightness and position within $50 \mathrm{~s}$.

5. The intensities of $\mathrm{Fe} I$ and $\mathrm{Fe}$ II lines under study are higher in polar facular points than in the ambient atmosphere, throughout the line profiles. Yet relative to the local continuum, the $\mathrm{Fe}_{\mathrm{I}}$ lines from $\mathrm{PFe}$ and surroundings are similar, while the Fe II 6149.3 line is stronger in PFe.

6. Strong Stokes $V$ signals of up to $\left|V_{\text {extr }}\right|=0.06 \times I_{\mathrm{c}}$ are found in $\mathrm{Fe}$ I 6302.5 which requires high area filling factors up to $f \approx 0.6$ and strong, kilo-Gauss fields.

7. $\mathrm{PFe}$ are unipolar magnetic features, they possess the same magnetic polarity as the global magnetic field. We thus confirm the result by Homann et al. (1997).

8. We find an average upward flow of the plasma within the magnetic flux tubes of PFe of $v=0.5-1 \mathrm{~km} \mathrm{~s}^{-1}$, relative to rest on the solar surface, albeit with a large scatter reflecting the intrinsic, highly dynamic PF properties.

These results on PFe need further observational confirmation and should be contrasted with data from equatorial faculae when observed also near the limb. They also give information 
on size, intensity, polarimetric properties and line shifts and will serve as constraints for the modelling of PFe.

Acknowledgements. We thank Dr. J. Sánchez Almeida for his fruitful comments on a draft version of this publication. Part of this work was supported by the German Academic Exchange Service $D A A D$ through grant A/00/01395. The Gregory-Coudé Telescope had been operated by the Universitäts-Sternwarte Göttingen at the Spanish Observatorio del Teide of the Instituto de Astrofísica de Canarias. It was dismounted in spring 2002. The Vacuum Tower Telescope is operated by the Kiepenheuer-Institut für Sonnenphysik, Freiburg, at the Observatorio del Teide.

\section{References}

Auffret, H., \& Muller, R. 1991, A\&A, 246, 264

Bendlin, C., \& Volkmer, R. 1995, A\&AS, 112, 371

Bendlin, C., Volkmer, R., \& Kneer, F. 1992, A\&A, 257, 817

Brault, J. W., \& White, O. R. 1971, A\&A, 13, 169

Choudhuri, A. R., Auffret, H., \& Priest, E. R. 1993, Sol. Phys., 143, 49

de Boer, C. R. 1996, A\&AS, 120, 195

DeForest, C. E., Hoeksema, J. T., Gurman, J. B., et al. 1997, Sol. Phys., 175, 393

Deinzer, W., Hensler, G., Schüssler, M., \& Weisshaar, E. 1984a, A\&A, 139,426

Deinzer, W., Hensler, G., Schüssler, M., \& Weisshaar, E. 1984b, A\&A, 139,435

Domínguez Cerdeña, I., Kneer, F., \& Sánchez Almeida, J. 2003, ApJ, 582 , L55

Emonet, T., \& Cattaneo, F. 2001, ApJ, 560, L197

Hirzberger, J., Koschinsky, M., Kneer, F., \& Ritter, C. 2001, A\&A, 367,1011

Homann, T., Kneer, F., \& Makarov, V. I. 1997, Sol. Phys., 175, 81

Janßen, K. 2003, Ph.D. Thesis, Göttingen university

Janßen, K., Vögler, A., \& Kneer, F. 2003, A\&A, 409, 1127

Joliffe, I. T. 1986, Principal Component Analysis (New York: Springer)

Keller, C. U., \& von der Lühe, O. 1992, A\&A, 261, 321

Koschinsky, M., \& Kneer, F. 1996, A\&AS, 119, 171

Koschinsky, M., Kneer, F., \& Hirzberger, J. 2001, A\&A, 365, 588
Krieg, J., Wunnenberg, M., Kneer, F., Koschinsky, M., \& Ritter, C. 1999, A\&A, 343, 983

Landi Degl'Innocenti, E. 1992, in Solar Observations: Techniches and interpretations, ed. F. Sánchez, M. Collados, \& M. Vázquez (Cambridge: Cambridge University Press), 71

Lawrence, J. K., \& Chapman, G. A. 1988, ApJ, 335, 996

Makarov, V. I., \& Sivaraman, K. R. 1989, Sol. Phys., 123, 367

Makarov, V. I., Tlatov, A. G., \& Sivaraman, K. R. 2003a, Astron. Nachr., 324, 382

Makarov, V. I., Tlatov, A. G., \& Sivaraman, K. R. 2003b, Sol. Phys., 214, 41

Marsch, E. 1998, Nonlinear Processes in Geophys., 5, 111

Marsch, E., \& Tu, C.-Y. 1997, A\&A, 319, L17

Neckel, H. 1999, Sol. Phys., 184, 421

Okunev, O. V. 2004, Ph.D. Thesis, Göttingen university, in preparation

Ortiz, A., Solanki, S. K., Domingo, V., Fligge, M., \& Sanahuja, B. 2002, A\&A, 388, 1036

Pérez Rodríguez, E., \& Kneer, F. 2002, A\&A, 395, 279

Rouppe van der Voort, L. H. M., Rutten, R. J., Sütterlin, P., Sloover, P. J., \& Krijger, J. M. 2003, A\&A, 403, 277

Sánchez Almeida, J., \& Lites, B. W. 2000, ApJ, 532, 121

Sánchez Almeida, J., Martínez Pillet, V., \& Kneer, F. 1995, A\&AS, 113,359

Sánchez Cuberes, M., Vázquez, M., Bonet, J. A., \& Sobotka, M. 2002, ApJ, 570, 886

Schröter, E. H. 1956, ZAp, 41, 141

Sheeley, N. R., Jr. 1964, ApJ, 140, 731

Sheeley, N. R., Jr. 1991, ApJ, 374, 386

Stolpe, F., \& Kneer, F. 1998, A\&AS, 131, 181

Vernazza, J. E., Avrett, E. H., \& Loeser, R. 1981, ApJS, 45, 635

Vögler, A., \& Schüssler, M. 2003, Astron. Nachr., 324, 399

von der Lühe, O. 1984, J. Opt. Soc. Am. A1, 510

Waldmeier, M. 1955, ZAp, 38, 37

Waldmeier, M. 1962, ZAp, 54, 260

Wang, Y.-M., \& Sheeley, N. R., Jr. 2003, ApJ, 599, 1404

Weigelt, G. P. 1977, Optics Comm., 21, 55

Wellstein, S., Kneer, F., \& von Uexküll, M. 1998, A\&A, 335, 323

Wilhelm, K., Marsch, E., Dwivedi, B. N., et al. 1998, ApJ, 500, 1023

Wilhelm, K., Dammasch, I. E., Marsch, E., \& Hassler, D. M. 2000, A\&A, 353, 749

Xia, L., Marsch, E., \& Curdt, W. 2003, A\&A, 399, L5

Zhang, M., \& Zhang, H. O. 1999, A\&A, 352, 317 